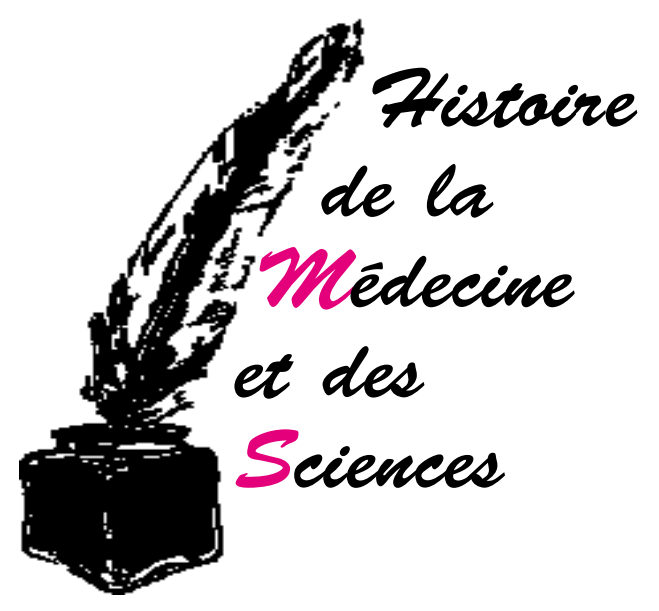

\title{
Les leucémies aiguës: une vue historique
des classifications une vue historique
des classifications
}

$\mathrm{L}$ es biologistes ont généralement admis que les classifications comportent aussi bien des éléments pragmatiques (étant formées en vue d'un but pratique) que des éléments rationnels (correspondant à une méthodologie de connaissance et à une visée théorique). Cette double orientation est manifeste en pathologie. En outre, un certain taux de cas inclassables y subsiste. Peut-on penser que le perfectionnement des classifications puisse aider à la connaissance des mécanismes physiopathologiques et, par-delà les mécanismes, à une connaissance plus étiologique des leucémies? Certains problèmes posés par ces classifications, comme la définition des types cellulaires pathologiques par rapport aux types normaux, et l'existence de formes mixtes, furent rencontrés par les pathologistes du XIX $\mathrm{X}^{\mathrm{e}}$ siècle qui, les premiers, étudièrent les leucémies. D'autres phénomènes qui compliquaient la tâche des classificateurs furent mis en évidence plus tard. Le hiatus leukaemicus nommé par Otto Naegeli à la fin du XIX ${ }^{\mathrm{e}}$ siècle désignait la coexistence dans le sang de cellules normales et pathologiques. Le mosaïcisme, sur lequel Marcel Bessis a insisté, désigne l'émergence de nouvelles populations différentes de cellules leucémiques.

Parmi les premiers observateurs qui décrirent les leucémies figure le médecin et microscopiste Alfred Donne en France [1]. Les leucémies rencontrées en 1845 par Bennett, qui proposa le nom de leucocytémie, et par Virchow, qui proposa celui de leucémie [2], étaient chroniques. Virchow les définit comme perturbations du développement des cellules sanguines, et distingua des formes splénique (avec atteinte de la rate) et lymphatique, mais il dut également reconnaître des formes mixtes, et il souligna la difficulté de définir les limites du concept de leucémie. La découverte de la fonction hématopoiétique de la moelle osseuse par Ernst Neumann en 1869 conduisit au concept de leucémie myélogène et à une classification en trois formes (myélogène pure, splénique ou lymphatique avec trouble de la moelle). Les colorations histologiques utilisées par Paul Ehrlich dans les années 1880 permirent de grands progrès dans la distinction des formes cellulaires mûres et dans l'étude de leurs précurseurs (myélocytes d'Ehrlich, myéloblastes de Naegeli) [3], et révolutionnèrent le diagnostic différentiel des leucémies par rapport aux leucocytoses ou aux diverses anémies sanguines. Ehrlich proposa une théorie dualiste de l'origine des lignées leucocytaires myéloïde et lymphoïde. Ce fut seulement en 1889 que Wilhelm Ebstein discuta des cas d'évolution rapide qu'il nomma leucémies aiguës et distingua de la transformation aiguë des leucémies chroniques. Les deux types pouvaient avoir des étiologies différentes. Le dualisme d'Ehrlich trouvait des arguments dans l'étude des leucémies : dans les leucémies lymphoïdes aiguës, on observe une substitution rapide du tissu lymphoïde au tissu myéloïde dans la moelle osseuse, avec disparition des myélocytes. Aux yeux d'Ehrlich, les leucémies lymphoïdes aiguës apportaient la preuve que les lymphocytes sont sans relation avec les polynucléaires. En 1898, Ehrlich et Lazarus distinguèrent leucémies myélogènes et leucémies lymphatiques et, à l'intérieur de ce dernier groupe, leucémies aiguës et chroniques avec des subdivisions possibles pour les leucémies chroniques. Ehrlich déclarait ignorer si la leucémie lymphatique chronique était une maladie unique ou si elle devait être subdivisée quant à l'étiologie [4].

Les leucémies lymphatiques aiguës furent identifiées avant les leucémies myéloïdes aiguës. La connaissance de ces dernières bénéficia de l'étude des types cellulaires de la moelle osseuse et de l'identification des myéloblastes, précurseurs des myélocytes, par Otto Naegeli qui les distingua des lymphocytes, distinction fondée sur des données morphologiques, cytochimiques et cliniques [5]. Naegeli décrivit la leucémie myéloblastique, entité qui se substituait naturellement au diagnostic du mélange de deux leucémies. A ses yeux toutes les leucémies myéloïdes aiguës étaient d'emblée myéloblastiques. Naegeli a fortement souligné la capacité des leucémies de se différencier en deux formes sans transition ni genèse unitaire. L'anatomie pathologique ne pouvait montrer de transformation de tissu lymphatique en myéloïde ou réciproquement [6]. Naegeli était 
fortement influencé par la pensée taxinomique insistant sur les discontinuités. La question de formes mixtes ou de transition fut débattue. Des cas de leucémie lymphatique aiguë décrits par Hans Hirschfeld montraient la prédominance des lymphocytes dans la moelle osseuse et la présence de nombreux myélocytes dans les ganglions lymphatiques, ce qui atténuait la distinction entre les lignées lymphocytaire et granulocytaire [7]. Contre le dualisme d'Ehrlich et de Naegeli, l'existence de formes mixtes orientait à nouveau vers l'idée d'une origine unique des cellules du sang.

Il est difficile de séparer classification et étiologie. Naegeli, qui cherchait à identifier différentes causes sous différentes formes, distingua les leucémies de diverses anémies, à ses yeux d'origine toxique ou infectieuse, et critiqua l'assimilation des leucémies lymphatiques à des cancers soutenue par Guido Banti [8] ou par Carl Sternberg. Pour Naegeli le caractère systémique des leucémies allait contre l'idée d'un processus néoplasique nécessairement local. Les leucémies devaient résulter d'un trouble de la régulation hormonale de la croissance tissulaire. Une preuve importante du caractère néoplasique des leucémies fut cependant donnée en 1934 par Jean Bernard qui provoqua une érythro-leucémie expérimentale par l'injection intramédullaire de goudron chez le rat [9]. Naegeli fut l'un des géants de l'hématologie de son temps. Jean Bernard a rapporté une discussion sur le myéloblaste entre Naegeli et un autre grand hématologiste, Adolfo Ferrata, au milieu des années trente. Naegeli mit fin à la discussion en disant: "A mon âge, vous ne changerez pas mes opinions " [10].

Contre Ehrlich et Naegeli, Arthur Pappenheim soutint une conception monophylétique de l'origine des cellules du sang et admit l'existence de précurseurs d'allure lymphoïde des lignées myéloïdes. Cela avait des conséquences pour la conception des leucémies. De nombreux cas de leucémies aiguës peuvent se réduire à des leucémies à grandes cellules

Dans ces leucémies aiguës atypiques, résultant d'une dédifférenciation des tissus lymphoïde et myéloïde, et présentant un tableau intermédiaire entre les formes typiques, toutes les espèces possibles de dissociation entre différenciation et maturation des cellules, ou entre maturation du noyau et du cytoplasme se rencontrent. Pappenheim prit au sérieux l'idée de pathologie cellulaire. Il chercha à établir une classification et une terminologie adéquates pour les cellules pathologiques et à élaborer une science nouvelle de la cytomorphogenèse [12]. Lui aussi uniciste, Adolfo Ferrata rechercha la cellule souche indifférenciée et la nomma hémocytoblaste (et plus tard hémohistioblaste) [13].

En 1916, Giovanni Di Guglielmo proposa une classification des leucémies aiguës fondée sur un accord total entre tableau sanguin et durée d'évolution. Il distinguait leucémie hyperaiguë ou hémocytoblastique (à hémocytoblastes prédominants), leucémie aiguë ou myélo- ou lymphoblastique, leucémie subaiguë ou à types cellulaires mélangés [14]. Commentant cette classification cytologique et son miraculeux parallélisme entre critères, Rieux en 1924 remarqua que certains auteurs admettaient l'unicité de la leucémie aiguë définie comme leucémie à cellules souches indifférenciées. Le caractère aigu était lié à une forte dédifférenciation. Le progrès des techniques de coloration amena Reschad et Schilling-Torgau à proposer en 1913 le terme de monocyte pour unifier des observations récentes sur de grands mononucléaires à fines granulations azurophiles [15] distingués par Aschoff et Kiyono des lymphocytes et des granulocytes [16]. Les monocytes furent reconnus comme troisième lignée leucocytaire indépendante (trialisme) donnant lieu à un troisième type de leucémie, la leucémie à monocytes, observée par Reschad et Schilling-Torgau. En raison de la similitude des monocytes et des histiocytes dans la phagocytose des colorants vitaux, Aschoff et Kiyono proposèrent en 1924 le concept de système réticulo-endothélial, et les observations sur le troisième type de leucé- mie, ou "réticulo-endothéliose», se multiplièrent. Pour Merklen et Wolff, la leucémie à monocytes pouvait exister sous trois formes, chronique, aiguë et inclassable (en raison du caractère évolutif du tableau sanguin) [17]. La question de la place des monocytes et des histiocytes fut très débattue. Ferrata faisait dériver directement le monocyte de la cellule souche, qu'il désignait comme l'hémohistioblaste. En 1930, William Dameshek concluait à la particularité de la leucémie monocytaire aiguë [18]. Certains auteurs lui attribuaient une origine infectieuse.

Avant la Deuxième Guerre mondiale, le parallélisme recherché par les classificateurs entre biologie et clinique fut rarement atteint. Le progrès vint plutôt de la morphologie et des colorations, et l'observation des leucémies valida pour l'essentiel des concepts morphologiques. Un certain chaos nosographique persistait dans la classification des maladies de l'appareil hématopoiétique à côté des leucémies. Lifschitz en 1930 ne séparait pas classification et étiologie. Il mentionnait les travaux d'Ellermann et Bang sur la transmission et l'étiologie virale ainsi que ceux de Peyton Rous. En 1938, Maurice Richter écrivait: "Tant que l'étiologie des leucémies est inconnue et que leurs relations mutuelles et à d'autres maladies restent obscures, toute tentative de classification doit être regardée comme un expédient temporaire $»[19]$.

A la suite de la Deuxième Guerre mondiale, la thérapeutique et le pronostic des leucémies s'améliorèrent, par l'exsanguino-transfusion pratiquée par Jean Bernard et Marcel Bessis en 1947, par la chimiothérapie à l'aminoptérine pratiquée par Sydney Farber en 1947 également. La thérapeutique modifiait le pronostic et rentrait dans les paramètres des classifications. La microscopie électronique établit la morphologie cellulaire sans détrôner les colorations. Les réactions cytochimiques fournissaient des tests pour l'identification des cellules pathologiques. A côté des données morphologiques, cytochimiques et thérapeutiques, la sensibilité des cultures cellulaires aux cytokines fut 
également introduite dans les classifications, avant les données immunologiques. La multiplication des critères ne favorisa pourtant pas leur concordance et un certain nombre de cas restèrent inclassables. En 1974, Marcel Bessis s'interrogeait sur la signification de l'inclassable [20]. Une cellule pathologique peut avoir perdu les caractéristiques qui permettent de l'identifier ou en avoir acquis certaines autres qui échappent à tout classement. Se demandant si des cellules d'origine lymphocytaire ne pouvaient pas acquérir des caractéristiques des myéloblastes, ou inversement, il faisait renaître l'idée de leucémie mixte. La progression $\mathrm{du}$ processus leucémique qui fait apparaître de nouvelles populations cellulaires fait elle-même obstacle à la classification.

Quatre regards ont été portés depuis les années 1970 sur les leucémies aiguës. Si tous visaient à définir des groupes permettant de comparer et d'améliorer les protocoles thérapeutiques, chacun a dessiné les contours de son objet en même temps que son questionnement. Les cytologistes prirent en charge la globalité du processus tumoral en individualisant les populations cellulaires majoritaires retrouvées dans la moelle osseuse et le sang. Les immunologistes, à l'aide d'anticorps monoclonaux détectant des antigènes membranaires, et d'études des réarrangements des gènes des immunoglobulines (Ig) et du récepteur des cellules $\mathrm{T}$ (TCR), ont eu le mérite de pouvoir affirmer la monoclonalité d'une population de cellules B ou T et d'ébaucher une caractérisation de son état de différenciation. Cette approche, qui a complété la précédente, a permis de proposer une place aux cellules normales ou tumorales à l'intérieur de schémas de différenciation lymphoïde ou (à un moindre degré) myéloïde. Les cytogénéticiens ont porté leur attention sur les chromosomes dont ils ont décrit les anomalies. Ils ont finalement guidé les biologistes moléculaires vers le clonage des régions impliquées. C'est avec ces derniers que la compréhension des leucémies a quitté le terrain morphologique et descriptif pour gagner celui des anomalies des fonctions de protéines jouant un rôle dans différents secteurs de la physiologie cellulaire.

Chacune de ces approches a engendré ses propres classifications qui continuent aujourd'hui à faire l'objet de confrontations. Au début des années 1970, les critères morphologiques permettaient de distinguer les leucémies aiguës lymphoblastiques, caractérisées par leur prédominance au cours de l'enfance, par leur réponse aux traitements, des autres leucémies regroupées sous le terme générique de leucémies myéloïdes aiguës, qui se subdivisaient en fonction du type cellulaire prédominant (leucémies myéloblastique, monocytaire, érythroblastique...) [21].

En même temps que des progrès thérapeutiques étaient enregistrés, une confusion s'est installée dans les termes utilisés pour désigner les cellules leucémiques. Cela rendait difficile la comparaison des résultats. Cette constatation a débouché sur la réunion d'un groupe de travail composé d'hématologistes français, américains et britanniques qui, à partir d'octobre 1974, ont confronté leurs opinions sur 200 cas de leucémies aiguës et d'affections pouvant poser des problèmes de diagnostics différentiels. Ces travaux ont conduit à la publication en 1976 de la classification $\mathrm{FAB}$ qui visait à établir deux distinctions [22]. La première consistait à définir des critères objectifs permettant de dissocier les leucémies myéloïdes des leucémies non myéloïdes ( lymphoblastiques»). Ces deux groupes furent ensuite subdivisés en trois formes de leucémies «lymphoblastiques" (LAL) (L1, L2, L3) et six formes de leucémies myéloïdes aiguës (LAM) (M1 à M6). L'immense avantage de cette classification, fondée sur l'examen de lames colorées et l'emploi de réactions cytochimiques (myéloperoxidases et estérases spécifiques), est d'avoir été rapidement acceptée et d'avoir offert une référence commune pour les essais thérapeutiques.

L'apport de l'immunologie a été déterminant pour la compréhension des LAL et leur séparation d'avec certaines formes de LAM. Après la distinction initiale opérée entre les lymphocytes B et les lymphocytes $\mathrm{T}$ grâce aux récepteurs des globules rouges de mouton et aux immunoglobulines membranaires de surface, l'introduction des anticorps monoclonaux a, d'une part, permis de prendre conscience de l'hétérogénéité des LAL et, d'autre part, de proposer pour celles-ci une classification tenant compte de la différenciation lymphoïde [23-26]. Si, en raison de l'absence de marqueurs spécifiques de malignité, l'immunologie laisse toute sa place à la cytologie, seule à même de découvrir des anomalies morphologiques caractéristiques, elle retrouve tout son intérêt quand elle affirme la monoclonalité et le stade de différenciation [27, 28]. Les leucémies biphénotypiques qui possèdent à la fois des marqueurs lymphoïdes et myélö̈des peuvent morphologiquement s'apparenter à des LAM peu différenciées ou à des LAL. Elles sont à l'heure actuelle en voie d'évaluation par un groupe de travail européen (EGIL) qui vise à définir les bases immunologiques de leur autonomie. La découverte des leucémies biphénotypiques peut, d'une certaine façon, être considérée comme un nouvel exemple d'un thème récurrent dans l'histoire des leucémies, celui des leucémies mixtes et de la difficulté de classer les leucémies.

Grâce aux progrès de ses techniques, la cytogénétique a, à partir des années 1970, créé son propre champ nosologique. En même temps qu'étaient individualisées certaines anomalies spécifiques comme la translocation t $(4 ; 11)$ qui caractérise une LAL frappant de très jeunes enfants, était brossé un tableau de l'ensemble des modifications clonales du caryotype, primaires ou secondaires, numériques ou structurales, observées au cours des leucémies. Si la translocation $\mathrm{t}(4 ; 11)$ correspond à des LA L1, L2 (LAL de la lignée B ou biphénotypique sur le plan immunologique), et si d'autres anomalies chromosomiques se rencontrent dans différents groupes morphologiques de LA (classification FAB), certaines ne sont décrites que dans des LA particulières. C'est la cas 
de la LA M3 caractérisée par la translocation $\mathrm{t}(15 ; 17)$ qui ne se voit que dans cette forme de leucémie impliquant le récepteur de l'acide $\alpha$-rétinoïque $(\operatorname{RAR} \alpha)$. La cytogénétique a enfin guidé les biologistes moléculaires pour cloner les gènes intervenant dans la leucémogenèse et retrouvés aux points de cassure des translocations chromosomiques [29]. La biologie moléculaire a non seulement permis de fournir des techniques diagnostiques sensibles (PCR, Southern blot), mais elle a surtout offert la possibilité de connaître les anomalies moléculaires en cause dans les leucémies et les mécanismes cellulaires perturbés. Deux types de translocations ont fourni les modèles autorisant un classement de ces anomalies moléculaires. Le premier correspond à la translocation $\mathrm{t}(9 ; 22)$ observée dans la leucémie myéloïde chronique. Elle provoque la fusion des gènes $B C R$ et $\mathrm{c}-A B L$ aboutissant à la synthèse d'un ARNm et d'une protéine chimérique Bcr-Abl. Ces fusions de gènes s'observent dans plusieurs LA, dont la LA M3 caractérisée par la synthèse du polypeptide de fusion Pml-RAR $\alpha$. Le deuxième modèle découle de l'analyse du lymphome de Burkitt au cours duquel les gènes des Ig sont rapprochés du protooncogène c-MYC qui, en conséquence, est activé. Cette dernière anomalie est l'apanage de la LA L3. D'autres gènes sont activés au cours de LAL T accompagnées de remaniements des gènes du TCR. Si les protéines produits des gènes activés ou les protéines chimériques peuvent avoir des fonctions cellulaires diverses, la transcription s'est avérée être souvent sollicitée étant donné la présence au sein de ces polypeptides de motifs structuraux de liaison à l'ADN déduits de la séquence de leurs gènes [30, 31].

En raison du découpage des disciplines, nous avons distingué quatre approches de la classification et de la compréhension des leucémies aiguës, mais il est clair que les cloisons sont loin d'être étanches et que les spécialistes de ces différents domaines ont étroitement collaboré pour bouleverser en vingt ans les idées que l'on pouvait se faire des leucémies. En même temps que les différentes classifications ont fourni un moyen de comprendre les leucémies, elles ont permis la constitution de groupes homogènes servant d'appui aux essais thérapeutiques, qui participent également à la validation de ces classes. Cependant, avec la description des mécanismes moléculaires en cause dans les leucémies, les questions étiologiques sont progressivement devenues souterraines. Dans les années 1970, en effet, bien que l'étiologie des leucémies restât inconnue, certains facteurs semblaient pouvoir être incriminés: hérédité, radiations, agents chimiques et virus. Avec l'apparition de nouveaux critères, immunologiques et génétiques, l'évolution des classifications des leucémies est allée dans le sens d'une réduction de l'inclassable. Il ne faut pourtant pas oublier que ces critères sont liés à certains moments d'un processus évolutif qui constitue la maladie. Le développement de nouveaux critères permettra-t-il l'élimination complète de l'inclassable? Les travaux sur les leucémies permettront-ils de passer d'une étude moléculaire des mécanismes physiopathologiques à une identification des étiologies et à une classification étiologique?

\section{RÉFÉRENCES}

1. Degos L. Un pionnier de la médecine scientifique, Alfred Donne. médecine/sciences $1995 ; 10$ : 1478-81.

2. Virchow R. Zur pathologischen Physiologie des Blutes. Arch Path Anat Klin Med $1849 ; 2: 587-98$.

3. Ehrlich P. Gesammelte Arbeiten. Berlin: Springer, 1956: vol. 1, 210-11.

4. Ibid., 251.

5. Naegeli O. Uber rothes Knochenmark und Myeloblasten. Deutsche medicinische Wochenschrift 1900; 26: 289-90.

6. Fabian E, Naegeli O, Schatiloff P. Beiträge zur Kenntnis der Leukämie. Virch Arch Path Anat Klin Med 1907; 190: 480.

7. Hirschfeld H. Weiteres zur Kenntnis der myeloiden Umwandlung. Berl Kli Woch $1906 ; \mathrm{n}^{\circ}$ 32: 1067 .
8. Banti G. Die Leukämieen. Zentralblatt für allgemeine Pathologie und opathologische Anatomie 1904; 15 : 7-8.

9. Bernard J. Études anatomiques sur les syndromes érythro-leucémiques provoqués chez le rat par le goudron. Le Sang 1934; 8 : 992.

10. Bernard J. Introduction. Blood Cells $1975 ; 1: 4$.

11. Pappenheim A. Grundriss der hämatologischen Diagnostik und praktischen Blutuntersuchung. Leipzig: Werner Klinkhardt, 1911: 178.

12. Pappenheim A. Morphologische Hämatologie. Leipzig, Werner Klinkhardt, 1919: 745.

13. Ferrata A, Reitano D. Sindromi istiocitemiche (emoistioblastiche). Haematologica 1923; 4: 385-93.

14. Di Guglielmo G. La leucemia acuta. Napoli, N. Jovene, 1916.

15. Reschad H, Schilling-Torgau V. Ueber eine neue Leukämie durch echte Uebergagsformen (Splenozytenleukämie) und ihre Bedeutung für die Selbstständigkeit dieser Zellen. Muench Medi Woch 1913; 50 : 1981.

16. Aschoff L, Kiyono M. Zur Frage der grossen Mononukleären. Folia Haematol $1913 ; 15: 383$.

17. Merklen P, Wolff M. Monocytes. Monocytoses. Leucémies à Monocytes. Presse Méd 1927; $35: 148$.

18. Dameshek W. Acute monocytic leukemia. Review of the literature and case reports. Arch Int Med 1930; 46: 718.

19. Richter M. Leucemia. In: Downey H, ed. Handbook of hematology, vol. 4. New York: Paul B. Hoeber, 1938: 2887.

20. Bessis M. Pathology of the leukemic cell or reasons why some leukemia are unclassifiable. Blood Cells 1975; 1: 186.

21. Kay HEM. The acute leukemias. In: Hardity RM, Weatherall DJ, eds. Blood and its disorders. Oxford, London, Edinburgh, Melbourne: Blackwell Scientific Publications, 1974: 900 .

22. Bennett JM, Catovsky D, Daniel MT, et al. Proposals for the classification of acute leukemias. Br J Haematol 1976 ; 33: 451.

23. Foon KA, Schroff RW, Gale RP. Surface Markers on leukemia and lymphoma cells : recent advances. Blood 1982; 60: 1 .

24. Foon KA, Todd RF. Immunologic classification of leukemia and lymphoma. Blood $1986 ; 68: 1$.

25. Nadler LM, Korsmeyer SJ, Anderson $\mathrm{KC}$, et al. B cell origin of non-T cell acute lymphoblastic leukemia. A model for discrete stages of neoplastic and normal pre-B cell differentiation. J Clin Invest 1984; 74: 332. 


\section{RÉFÉRENCES}

26. Griffin JD, Mayer RJ, Weinstein HJ, et al. Surface marker analysis of acute myeloblastic leukemia : Identification of differentiationassociated phenotypes. Blood 1983 ; 62 :

557.

27. Bernard A. Classification immunologique des leucémies aiguës lymphoblastiques.

In Dreyfus B, Hématologie. Paris :

Flammarion, 1992 : 771.

28. Merle-Béral H. Classification immunologique des leucémies aiguës myéloïdes. In

Dreyfus B. Hématologie. Paris : Flammarion, 1992 : 776.

29. Tanzer J, Huret JL. Cytogénétique des Hémopathies malignes. In : Dreyfus B,

Hématologie. Paris : Flammarion, MédecineSciences, 1992 : 314.

30. Rabbits TH. Chromosomal translocations in human cancer. Nature 1994 ; 372 :

143.

31. Cline MJ. The molecular basis of leukemia. N Eng J Med 1994 ; 330 : 328.

\section{Remerciements}

Les auteurs tiennent à remercier le Professeur

Jean Bernard pour ses discussions et ses remarques.

\section{Claude Debru}

Centre Européen d'Histoire de la Médecine, Faculté de Médecine, 4, rue Kirschleger, 67085 Strasbourg Cedex, France.

\section{Patrick Triadou}

Laboratoire Central d'Hématologie, Groupe Hospitalier Necker-Enfants Malades,

149, rue de Sèvres, 75015 Paris, France.

\section{TIRÉS À PART}

C. Debru 